\title{
Metastatic melanoma: can FDG-PET predict success of anti-PD-1 therapy and help determine when it can be discontinued?
}

\author{
Elif Hindié ${ }^{1,2}$ \\ Published online: 22 April 2020 \\ (C) Springer-Verlag GmbH Germany, part of Springer Nature 2020
}

Immune checkpoint inhibitors (ICIs) that revive the immune system have revolutionized the management of advanced melanoma. Ipilimumab, an antibody aiming CTLA-4 (cytotoxic T lymphocyte associated antigen 4), was the first immunotherapy to increase overall survival (OS) [1]. Antibodies directed against PD-1 (programmed cell death 1) offered higher efficacy $[2,3]$. In KEYNOTE-006 trial, median OS was 32.7 months with pembrolizumab and 15.9 months with ipilimumab (hazard ratio (HR) $0.73 ; p=0.00049$ ) [2]. PD-1 antibodies are characterized by long-lasting responses $[2,3]$. However, many patients do not derive benefit. For example, although median OS with nivolumab was 37.5 months, the OS rate at 1 year was $71 \%$ [3]. In addition, severe immune-related side effects (grade $\geq 3$ ) occur in about $15 \%$ of patients $[2,3]$. They are unpredictable and often occur within the early months. Biomarkers predictive of the benefit (or lack thereof) from anti-PD-1 therapy are currently lacking. Tumor expression of PD-L1 (programmed death-ligand 1) by immunohistochemistry has only weak predictive value [4]. By assessing heterogeneity in expression, whole-body PD-L1 imaging might prove valuable in the future [5]. Many other biomarkers are being explored, such as the density and types of CD8+ T cells or the co-occurrence of tumor-associated $\mathrm{CD} 8+\mathrm{T}$ cells and CD20+ B cells [6]; tumor mutational burden; circulating tumor DNA [7]; the derived neutrophil-to-lymphocyte ratio (dNLR), alone or in combination with lactate dehydrogenase (LDH) level $[8,9]$; and specific characteristics of the composition of gut microbiota [10]. However, none of these biomarkers has been clinically validated as a factor for patient selection.

This article is part of the Topical Collection on Oncology - Chest

Elif Hindié

elif.hindie@chu-bordeaux.fr

1 Department of Nuclear Medicine, Bordeaux University and Hospitals, Bordeaux, France

2 Hôpital Haut-Lévêque, Avenue Magellan, 33604 Pessac, France
Moreover, due to the induced immunological flare in tumoral lesions and in non-tumoral tissues (including sarcoid reactions), these novel treatments have brought significant complexity to the interpretation of early therapeutic response, either by morphological or by metabolic imaging [11-14]. While early imaging can identify some early responders or help depict immunotherapy-related side effects [13], it is unable to differentiate true progressive disease from "pseudoprogression," which is characterized by an increase in the size of lesions or the visualization of new lesions, followed by a response [14]. These patients have an intermediate prognosis [11]. It has been considered that conventional RECIST v1.1 criteria might underestimate the benefit from anti-PD-1 therapy in up to $15 \%$ of patients with advanced melanoma [11]. Modified imaging criteria have been developed, such as immune-related response criteria and iRECIST [11, 15]. They introduce the concept of "unconfirmed progression" which allows to continue treatment in the absence of deteriorating clinical parameter, unless further progression is documented on later imaging, performed at least 4 weeks after initial evaluation $[15,16]$. However, for patients who end up having true progression, there is a risk of precious time lost and additional treatment toxicity. Difficulties in early response assessment in melanoma have been documented [17]. In one study, neither of RECIST 1.1, immune-related response, PERCIST, or EORTC criteria demonstrated sufficient accuracy [17]. 18F-FDG PET/CT offered better accuracy at 4 months from the start of treatment, and with the use of some modified response criteria [18-20].

\section{Association between baseline 18F-FDG $\mathrm{PET} / \mathrm{CT}$ imaging and outcomes under ICI therapy}

18F-FDG PET/CT is used in many institutions for assessing disease extent in patients with advanced melanoma [21]. In this issue of the journal, Seban and colleagues investigated 
whether imaging biomarkers derived from a quantitative analysis of baseline 18F-FDG PET/CT have prognostic value in patients treated with immune checkpoint inhibitors [22]. They retrospectively studied 32 patients with cutaneous melanoma treated with PD-1 antibodies and 24 patients with mucosal melanoma treated either with ipilimumab or with PD-1 antibodies at Gustave Roussy Comprehensive Cancer Center, France [22].

As regards patients with mucosal melanoma (head and neck or esophageal, vulvo-vaginal, anorectal), the median OS was 23.9 months. Higher baseline tumor SUVmax was associated with shorter OS (adjusted $p=0.02$ ), while volumebased PET parameters were not. An important information in this rare entity is that SUVmax values (median 12.5) allowed appropriate tumor delineation in all patients [22]. Thus, FDGPET can be used for assessment of disease extension in a similar way as it is used in cutaneous melanoma [21]. The association between baseline SUVmax and outcomes under ICI therapy is an interesting observation that deserves investigation in prospective studies for confirmation and to see how this information can be used for patient management. The overall response to ICI monotherapy was rather low, as in other series with mucosal melanoma [23, 24], with $58 \%$ of patients having progressive disease as their best response (compared with $31 \%$ in patients with cutaneous melanoma) [22]. Forschner and colleagues reported on hyperprogression under ICI occurring in a non-negligible percentage of patients, notably in anorectal melanoma [24]. Hyperprogression under ICI has been less described in classical cutaneous melanoma, while it is well recognized in some other solid cancers, such as non-small cell lung cancer [25].

The findings reported by Seban and colleagues in patients with cutaneous melanoma, uniformly treated with anti-PD-1 monotherapy, are of major importance [22]. Thirty-one percent of patients had progressive disease as best response (disease control rate 69\%). Median progression-free survival (PFS) was 10.7 months, and the median OS was 28.3 months. SUVmax was not associated with outcomes, while the total metabolic tumor volume (TMTV) was significantly associated with disease control rate $(p=0.04)$, PFS (adjusted $p=$ 0.01 ), and OS (adjusted $p=0.01$ ) [22]. Another parameter, BLR (bone marrow-to-liver SUVmax ratio; bone marrow uptake being measured in L1 to L4), was also associated with disease control rate $(p=0.04)$, PFS (adjusted $p=0.03$ ), and OS (adjusted $p=0.04$ ). Correlation between TMTV and BLR was low; these PET parameters were independently associated with outcomes [22]. In a previous study, in which Seban and colleagues exploited data of 55 patients from two centers, TMTV and BLR, with cutoffs corresponding to their median values, were combined to yield three groups of patients with significantly different prognoses. Median OS were 13.9 months for the high-risk group (TMTV $>25 \mathrm{~cm}^{3}$ and BLR $>0.79 ; n=18$ patients), 36.7 months for the intermediate-risk group $(n=19)$, and 52.4 months for the low-risk group (TMTV $>25 \mathrm{~cm}^{3}$ and BLR $>0.79 ; n=19$ ) [26]. One limitation is that many patients had received prior systemic treatments or radiotherapy $[22,26]$, which might have an impact on BLR. It will be important to confirm the findings in series of first-line anti-PD-1 therapy and to assess the value of BLR compared with other biomarkers, such as dNLR or other inflammatory markers [8]. Larger series would allow for multivariate analyses that take into account not only PET parameters but also some well-known prognostic factors in advanced cutaneous melanoma, such as sites of metastatic disease (i.e., the subcategories M1a, M1b, M1c, and M1d), LDH level [27], age, and functional status. Also, difficulties in delineating brain metastases on FDG-PET, and whether these patients should be excluded from FDG-PET-based predictive models, need to be clarified.

Baseline tumor size, as assessed by summing up the longest dimensions of target lesions on morphological imaging, has also been seen to be correlated with outcome under antiPD-1 therapy [28]. TMTV on whole-body PET is a better measure of tumor burden as it is not limited to 5 target lesions. It is easy to obtain and showed good reproducibility between readers in many studies [29]. However, for proposed prognostic cutoffs to be reproducible between centers, standardization is needed, notably in methods used for volume segmentation.

Ito and colleagues, from the Memorial Sloan Kettering Cancer Center in New York, USA, were the first to report on the prognostic value of baseline TMTV in melanoma patients receiving ICI [30]. The study comprised 142 patients (cutaneous melanomas and other entities) treated with ipilimumab. Patients with only brain metastases, or those in whom no tumor lesion could be delineated on FDG-PET, were not included. Baseline TMTV was significantly associated with OS while the intensity of FDG uptake (SULmax) was not. The median value for TMTV was $26.85 \mathrm{~cm}^{3}$. The median OS in patients with high TMTV was 10.84 months as compared with 26.09 months in patients with TMTV below the median ( $p=$ $0.002)$. TMTV remained significant in a multivariate analysis (HR 1.845; $p=0.007$ ) that also identified four significant clinical parameters (age $\geq 75$ years, previous chemotherapy, LDH above normal, active brain metastases). When combining TMTV with any of these clinical parameters, prognostic stratification was improved, leading to high-, intermediate-, and low-risk groups. Interestingly, while LDH level is to some degree a reflect of tumor burden, combining TMTV and LDH reinforced prediction. Patients with TMTV above median and elevated LDH $(n=30)$ had a median OS of 6.8 months, as compared with 52.47 months in patients $(n=56)$ with TMTV below median and LDH within normal [30].

The prognostic value of baseline TMTV in the setting of ICI therapy has also been documented in other solid tumors $[25,31]$. In non-small cell lung carcinoma (NSCLC), Seban and colleagues showed the value of combining this FDG-PET 
parameter with dNLR [31]. It will be important to distinguish the prognostic value of TMTV from its specific predictive value regarding anti-PD-1 therapy. For example, the lung immune prognostic index (stratifies patients based on dNLR greater than 3 and LDH above upper limit of normal as prognostic factors) was proposed as a simple blood-based biomarker to identify NSCLC patients unlikely to respond to ICI [32], and others found this prognostic index to also predict outcomes under ICI in melanoma and renal cell carcinoma [9]. However, this biomarker showed similar prognostic value in NSCLC patients treated with targeted therapies or chemotherapy, meaning that more work is needed to see how it can be used for treatment selection [33].

\section{Can baseline 18FDG parameters help selecting the most adequate initial therapy in patients with metastatic cutaneous melanoma?}

About $45 \%$ of patients with cutaneous melanoma carry an activating BRAF-V600 mutation. A major difficulty in current practice is the choice between first-line immunotherapy or a first-line treatment with BRAF/MEK inhibitor combination therapy (i.e., dabrafenib/trametinib, encorafenib/binimetinib, or vemurafenib/cobimetinib) [34]. These strategies have not been directly compared. A comparison between trials of the mean PFS and OS curves revealed a superiority of combined BRAF plus MEK inhibition within the first 12 months, later changing to a superiority of PD-1 blockers [35]. ESMO guidelines suggest that: "Patients for whom immunotherapy can be delivered safely for the first few months, i.e. patients with tumors not progressing very quickly and not immediately threatening an important organ or function, should be considered for immunotherapy first, preserving targeted therapies for the subsequent lines." [36]. ASCO guidelines give no preference between the two options [37]. If TMTV proves useful (alone or in combination with other metabolic, clinical, or biological parameters) in identifying patients who are unlikely to respond to anti-PD-1 therapy, then a strategy starting with BRAF plus MEK inhibition would be the best option for these patients. Regardless of whether TMTV is specifically predictive of outcomes under PD-1 blockers or generally prognostic, the benefit from starting with BRAF and MEK inhibitors in high-risk patients is that initial response rates are higher than with PD-1 blockers and these responses are of more rapid onset and less blurred by pseudo-progression phenomena, meaning that poor responders can be identified early. The use of short-term BRAF/MEK inhibitors as a bridge to anti-PD-1, or to anti-PD-1 anti-CTLA-4 combination therapy, might also be an option. This is one of the strategies explored in one trial investigating various sequencing strategies in unselected $B R A F$-mutated patients [38].

On the other hand, and regardless of BRAF-V600 status, combining anti-PD-1 and anti-CTLA-4 is one of the first-line options in metastatic melanoma [37]. In CheckMate 067, a descriptive analysis of OS data showed a trend towards superiority of the combination of nivolumab with ipilimumab, but no significance, in comparison with nivolumab monotherapy [4]. This combination is, however, associated with the highest rates of toxicity, with treatment-related grades 3-4 adverse events occurring in 59\% of patients [4]. Therefore, biomarkers are needed to better select patients that benefit from the combination (e.g., the presence of asymptomatic brain metastases) [36]. TMTV might prove useful in selecting high-risk patients to be offered combination therapy.

\section{Can 18FDG-PET/CT help determine if anti-PD-1 therapy can be discontinued safely?}

An equally important question is the optimal duration of antiPD-1 treatment in non-progressive patients. One of the main advantages with anti-PD-1 blockers, and more generally with immunotherapy, is that responses can be durable even after discontinuation, while targeted therapies are usually continued indefinitely in the absence of disease progression [1-4, 34]. However, as of yet, the optimal duration of anti-PD-1 therapy for metastatic melanoma remains unestablished [37]. In the relevant randomized trials, pembrolizumab was given for 2 years [2] and nivolumab could be continued beyond 2 years [4]. However, shorter courses of therapy can also be effective in properly selected patients. Unnecessarily long treatment duration may result in increased toxicity and place financial strain on health care systems [39].

Many studies have shown that patients with complete response (CR) have low rates of relapse after discontinuation of anti-PD-1 treatment. Settings and results are, however, heterogeneous [2, 40, 41]. In KEYNOTE-006, among patients who completed 2 years of pembrolizumab, 21 had a CR. At follow-up, the 24-month PFS was $85 \cdot 4 \%$ [2]. In the phase Ib KEYNOTE-001 study, $16.0 \%$ of patients treated with pembrolizumab achieved CR after a median time of 12 months. Among them, 67 patients elected to proceed with expectant observation (median time receiving pembrolizumab after CR was 7 months). The 24-month PFS rate from discontinuation was $89.9 \%$ [40]. In one large retrospective study, CR was seen in $25.8 \%$ of patients (102 of 396), but the definition of CR was more flexible than in clinical trials. The median total duration of treatment of these CR patients was 9.4 months, and the median duration of treatment after achieving a $\mathrm{CR}$ was 0 month (range, stopped before CR to 26 months after CR). PFS at 3 years from the date of CR was $72.1 \%$ [41]. 
The outcomes after discontinuation of anti-PD-1 treatment has also been studied in patients with partial response (PR) [2, 42]. In KEYNOTE-006, among patients who completed 2 years of pembrolizumab, there were 69 patients who achieved PR, while 13 had stable disease (SD). Estimated 24-month PFS was $82.3 \%$ for patients with PR as compared with $39.9 \%$ for patients with SD [2]. A large retrospective multicentric study investigated the outcome of patients who electively discontinued anti-PD-1 therapy with pembrolizumab or nivolumab outside clinical trials [42]. After a median follow-up of 18 months from treatment discontinuation, the risk of progression among patients with $\mathrm{CR}$ $(n=117)$ was $14 \%$ (with higher relative risk in patients treated for $<6$ months), while its was $32 \%$ among patients $(n=44)$ with PR [42]. The probability of response to retreatment with an anti-PD-1 was low (32\%) [42].

The data across these studies support that the majority of patients who achieve a CR with an anti-PD-1 blocker, but also many of those with PR, have good outcomes after treatment discontinuation. However, a means to improve selection is needed, notably as regards the opportunity of therapy discontinuation before 2 years. In this context, an important retrospective analysis from Melanoma Institute Australia investigated whether 18F-FDG PET can better predict long-term outcomes compared with CT-based response criteria [43]. Patients $(n=104)$ with metastatic melanoma had received FDG-PET and CT imaging at baseline and at 1 year after the start of ICI (anti-PD-1 therapy alone in $67 \%$ or combined with ipilimumab). The study did not include patients who have progressed at 1 year on CT by RECIST or on clinical grounds [43]. On CT at 1 year, 28\% had CR, 66\% had PR, and $6 \%$ had SD. Importantly, compete metabolic response (CMR) by EORTC criteria was observed in $68 \%$ of patients with PR on CT. PFS was significantly improved in patients with $\mathrm{PR}+\mathrm{CMR}$ versus PR + non-CMR (median not reached versus 12.8 month; HR $0.07 ; p<0.01$ ), with $100 \%$ versus $58 \%$ progression-free at 1 year, and $96 \%$ versus $48 \%$ progression-free at 2 years post imaging [43]. Overall, among the $78 \mathrm{CMR}$ patients (regardless of CT status), $78 \%$ had discontinued treatment with median follow-up post discontinuation of 14.5 months; 75 (96\%) remained progression-free [43]. Although the follow-up is still short, these findings suggest that in many patients with $\mathrm{PR}$, residual lesions on CT may represent scarring. Thus, additional assessment by FDG-PET might be very valuable to help determine the depth of response and guide clinical decisions, notably to inform on the possibilities of earlier treatment cessation than the arbitrary 2-year course in patients with CR, but also those with PR on CT but CMR on FDG-PET, or on the need to continue treatment beyond 2 years in patients with $\mathrm{PR}$ or SD with residual activity on FDG-PET.

\section{Concluding remarks}

The identification of patients with metastatic cutaneous melanoma who are unlikely to respond to anti-PD-1 therapy is an unmet need. Early assessment of response on morphological or metabolic imaging is unlikely to be the answer due to difficulties in differentiating true progressive disease from a pseudo-progression, unless it is used in combination with other biomarkers. However, as demonstrated in ipilimumabtreated patients and now in patients with PD-1 blockers, tumor burden defined by TMTV on baseline 18F-FDG PET/CT can identify patients who are unlikely to respond to ICI monotherapy. This parameter, alone or in combination with other metabolic, clinical, or biological parameters, could then be helpful for selecting alternative first-line options such as BRAF and MEK inhibition in $B R A F$-mutated patients or a combination of ICI regardless of $B R A F$ status. The predictive value of TMTV merits to be tested in prospective trials, where it can be used as a stratification factor. Limitations on FDG-PET imaging regarding assessment of brain metastases need to be recognized. 18F-FDG PET/CT can also be helpful for decisions regarding the safe interruption of anti-PD-1 treatments in patients with CR or PR on morphological imaging. Again, prospective trials would be very helpful for validation.

\section{Compliance with ethical standards}

Conflict of interest The author declares no conflict of interest.

\section{References}

1. Hodi FS, O'Day SJ, McDermott DF, Weber RW, Sosman JA, Haanen JB, et al. Improved survival with ipilimumab in patients with metastatic melanoma. N Engl J Med. 2010;363:711-23 Erratum in: N Engl J Med. 2010;363:1290.

2. Robert C, Ribas A, Schachter J, Arance A, Grob JJ, Mortier L, et al. Pembrolizumab versus ipilimumab in advanced melanoma (KEYNOTE-006): post-hoc 5-year results from an open-label, multicentre, randomised, controlled, phase 3 study. Lancet Oncol. 2019;20:1239-51.

3. Ascierto PA, Long GV, Robert C, Brady B, Dutriaux C, Di Giacomo AM, et al. Survival outcomes in patients with previously untreated BRAF wild-type advanced melanoma treated with nivolumab therapy: three-year follow-up of a randomized phase 3 trial. JAMA Oncol. 2019;5:187-94 Erratum in: JAMA Oncol. 2019;5:271.

4. Hodi FS, Chiarion-Sileni V, Gonzalez R, Grob JJ, Rutkowski P, Cowey CL, et al. Nivolumab plus ipilimumab or nivolumab alone versus ipilimumab alone in advanced melanoma (CheckMate 067): 4-year outcomes of a multicentre, randomised, phase 3 trial. Lancet Oncol. 2018;19:1480-92 Erratum in: Lancet Oncol. 2018;19:e668. Lancet Oncol. 2018;19:e581.

5. Bensch F, van der Veen EL, Lub-de Hooge MN, Jorritsma-Smit A, Boellaard R, Kok IC, et al. ${ }^{89} \mathrm{Zr}$-atezolizumab imaging as a noninvasive approach to assess clinical response to PD-L1 blockade in cancer. Nat Med. 2018;24:1852-8. 
6. Cabrita R, Lauss M, Sanna A, Donia M, Skaarup Larsen M, Mitra $\mathrm{S}$, et al. Tertiary lymphoid structures improve immunotherapy and survival in melanoma. Nature. 2020;577:561-5.

7. Forschner A, Battke F, Hadaschik D, Schulze M, Weißgraeber S, Han CT, et al. Tumor mutation burden and circulating tumor DNA in combined CTLA-4 and PD-1 antibody therapy in metastatic melanoma-results of a prospective biomarker study. J Immunother Cancer. 2019;7:180

8. Capone M, Giannarelli D, Mallardo D, Madonna G, Festino L, Grimaldi AM, et al. Baseline neutrophil-to-lymphocyte ratio (NLR) and derived NLR could predict overall survival in patients with advanced melanoma treated with nivolumab. J Immunother Cancer. 2018;6:74.

9. Meyers DE, Stukalin I, Vallerand IA, Lewinson RT, Suo A, Dean $\mathrm{M}$, et al. The lung immune prognostic index discriminates survival outcomes in patients with solid tumors treated with immune checkpoint inhibitors. Cancers (Basel). 2019;11(11):E1713. https://doi. org/10.3390/cancers 11111713 .

10. Gopalakrishnan V, Spencer CN, Nezi L, Reuben A, Andrews MC, Karpinets TV, et al. Gut microbiome modulates response to antiPD-1 immunotherapy in melanoma patients. Science. 2018;359: 97-103.

11. Hodi FS, Hwu WJ, Kefford R, Weber JS, Daud A, Hamid O, et al. Evaluation of immune-related response criteria and RECIST v1.1 in patients with advanced melanoma treated with pembrolizumab. J Clin Oncol. 2016;34:1510-7.

12. Borcoman E, Kanjanapan Y, Champiat S, Kato S, Servois V, Kurzrock R, et al. Novel patterns of response under immunotherapy. Ann Oncol. 2019;30:385-96.

13. Aide N, Hicks RJ, Le Tourneau C, Lheureux S, Fanti S, Lopci E. FDG PET/CT for assessing tumour response to immunotherapy: report on the EANM symposium on immune modulation and recent review of the literature. Eur J Nucl Med Mol Imaging. 2019;46: 238-50.

14. Humbert O, Cadour N, Paquet M, Schiappa R, Poudenx M, Chardin D, et al. ${ }^{18}$ FDG PET/CT in the early assessment of nonsmall cell lung cancer response to immunotherapy: frequency and clinical significance of atypical evolutive patterns. Eur J Nucl Med Mol Imaging. 2020;47:1158-67.

15. Seymour L, Bogaerts J, Perrone A, Ford R, Schwartz LH, Mandrekar S, et al. iRECIST: guidelines for response criteria for use in trials testing immunotherapeutics. Lancet Oncol. 2017;18(3): e143-52 Erratum in: Lancet Oncol. 2019;20:e242.

16. Beaver JA, Hazarika M, Mulkey F, Mushti S, Chen H, He K, et al. Patients with melanoma treated with an anti-PD-1 antibody beyond RECIST progression: a US Food and Drug Administration pooled analysis. Lancet Oncol. 2018;19:229-39.

17. Cho SY, Lipson EJ, Im HJ, Rowe SP, Gonzalez EM, Blackford A, et al. Prediction of response to immune checkpoint inhibitor therapy using early-time-point ${ }^{18} \mathrm{~F}$-FDG PET/CT imaging in patients with advanced melanoma. J Nucl Med. 2017;58:1421-8.

18. Anwar H, Sachpekidis C, Winkler J, Kopp-Schneider A, Haberkorn $\mathrm{U}$, Hassel JC, et al. Absolute number of new lesions on ${ }^{18} \mathrm{~F}-\mathrm{FDG}$ $\mathrm{PET} / \mathrm{CT}$ is more predictive of clinical response than SUV changes in metastatic melanoma patients receiving ipilimumab. Eur J Nucl Med Mol Imaging. 2018;45:376-83.

19. Ito K, Teng R, Schöder H, Humm JL, Ni A, Michaud L, et al. ${ }^{18} \mathrm{~F}$ FDG PET/CT for monitoring of ipilimumab therapy in patients with metastatic melanoma. J Nucl Med. 2019;60:335-41.

20. Annovazzi A, Vari S, Giannarelli D, Pasqualoni R, Sciuto R, Carpano S, et al. Comparison of 18F-FDG PET/CT criteria for the prediction of therapy response and clinical outcome in patients with metastatic melanoma treated with ipilimumab and PD-1 inhibitors. Clin Nucl Med. 2020;45:187-94.

21. Salaün PY, Abgral R, Malard O, Querellou-Lefranc S, Quere G, Wartski M, et al. Good clinical practice recommendations for the use of PET/CT in oncology. Eur J Nucl Med Mol Imaging. 2020;47:28-50.

22. Seban RD, Moya-Plana A, Antonios L, Yeh R, Marabelle A, Deutsch E, et al. Prognostic 18F-FDG PET biomarkers in metastatic mucosal and cutaneous melanoma treated with immune checkpoint inhibitors targeting PD-1 and CTLA-4. Eur J Nucl Med Mol Imaging. 2020. https://doi.org/10.1007/s00259-020-04757-3.

23. Nomura M, Oze I, Masuishi T, Yokota T, Satake H, Iwasawa S, et al. Multicenter prospective phase II trial of nivolumab in patients with unresectable or metastatic mucosal melanoma. Int J Clin Oncol. 2020. https://doi.org/10.1007/s10147-020-01618-9.

24. Forschner A, Hilke FJ, Bonzheim I, Gschwind A, Demidov G, Amaral T, et al. MDM2, MDM4 and EGFR amplifications and hyperprogression in metastatic acral and mucosal melanoma. Cancers (Basel). 2020;12(3).

25. Castello A, Rossi S, Toschi L, Mazziotti E, Lopci E. Hyperprogressive disease in patients with non-small cell lung cancer treated with checkpoint inhibitors: the role of ${ }^{18} \mathrm{~F}-\mathrm{FDG}$ PET/CT. J Nucl Med. 2019. https://doi.org/10.2967/jnumed.119.237768.

26. Seban RD, Nemer JS, Marabelle A, Yeh R, Deutsch E, Ammari S, et al. Prognostic and theranostic 18F-FDG PET biomarkers for antiPD1 immunotherapy in metastatic melanoma: association with outcome and transcriptomics. Eur J Nucl Med Mol Imaging. 2019;46: 2298-310.

27. Gershenwald JE, Scolyer RA, Hess KR, Sondak VK, Long GV, Ross MI, et al. Melanoma staging: evidence-based changes in the American Joint Committee on Cancer eighth edition cancer staging manual. CA Cancer J Clin. 2017;67:472-92.

28. Joseph RW, Elassaiss-Schaap J, Kefford R, Hwu WJ, Wolchok JD, Joshua AM, et al. Baseline tumor size is an independent prognostic factor for overall survival in patients with melanoma treated with pembrolizumab. Clin Cancer Res. 2018;24:4960-7 Erratum in: Clin Cancer Res. 2018;24:6098.

29. Vercellino L, Cottereau AS, Casasnovas RO, Tilly H, Feugier P, Chartier L, et al. High total metabolic tumor volume at baseline allows discrimination of survival even in patients aged 60 to 80 years responding to R-CHOP. Blood. 2020. https://doi.org/10. 1182/blood.2019003526.

30. Ito K, Schöder H, Teng R, Humm JL, Ni A, Wolchok JD, et al. Prognostic value of baseline metabolic tumor volume measured on ${ }^{18} \mathrm{~F}$-fluorodeoxyglucose positron emission tomography/computed tomography in melanoma patients treated with ipilimumab therapy. Eur J Nucl Med Mol Imaging. 2019;46:930-9.

31. Seban RD, Mezquita L, Berenbaum A, Dercle L, Botticella A, Le Pechoux C, et al. Baseline metabolic tumor burden on FDG PET/ CT scans predicts outcome in advanced NSCLC patients treated with immune checkpoint inhibitors. Eur J Nucl Med Mol Imaging. 2020;47:1147-57.

32. Mezquita L, Auclin E, Ferrara R, Charrier M, Remon J, Planchard $\mathrm{D}$, et al. Association of the lung immune prognostic index with immune checkpoint inhibitor outcomes in patients with advanced non-small cell lung cancer. JAMA Oncol. 2018;4:351-7.

33. Kazandjian D, Gong Y, Keegan P, Pazdur R, Blumenthal GM. Prognostic value of the lung immune prognostic index for patients treated for metastatic non-small cell lung cancer. JAMA Oncol. 2019;5:1481-5.

34. Robert C, Grob JJ, Stroyakovskiy D, Karaszewska B, Hauschild A, Levchenko E, et al. Five-year outcomes with dabrafenib plus trametinib in metastatic melanoma. N Engl J Med. 2019;381:62636 .

35. Ugurel S, Röhmel J, Ascierto PA, Becker JC, Flaherty KT, Grob JJ, et al. Survival of patients with advanced metastatic melanoma: the impact of MAP kinase pathway inhibition and immune checkpoint inhibition-update 2019. Eur J Cancer. 2020;130:126-38.

36. Michielin O, van Akkooi ACJ, Ascierto PA, Dummer R, Keilholz U, ESMO Guidelines Committee. Cutaneous melanoma: ESMO 
Clinical Practice Guidelines for diagnosis, treatment and followup $\dagger$. Ann Oncol. 2019;30:1884-901.

37. Seth R, Messersmith H, Kaur V, Kirkwood JM, Kudchadkar R, McQuade JL, et al. Systemic therapy for melanoma: ASCO guideline. J Clin Oncol. 2020:JCO2000198. https://doi.org/10.1200/ JCO.20.00198.

38. Ascierto PA, Dummer R, Melero I, Palmieri G, Giannarelli D, Abrami E, et al. SECOMBIT (sequential combo immuno and target therapy study): a three arms prospective, randomized phase II study to evaluate the best sequential approach with combo immunotherapy [ipilimumab (I) /nivolumab (N)] and combo target therapy [encorafenib (E)/binimetinib (B)] in patients with metastatic melanoma and BRAF mutation. J Clin Oncol. 2017;35(15_suppl): TPS9598.

39. Kandel M, Allayous C, Dalle S, Mortier L, Dalac S, Dutriaux C, et al. Update of survival and cost of metastatic melanoma with new drugs: estimations from the MelBase cohort. Eur J Cancer. 2018;105:33-40.

40. Robert C, Ribas A, Hamid O, Daud A, Wolchok JD, Joshua AM, et al. Durable complete response after discontinuation of pembrolizumab in patients with metastatic melanoma. J Clin Oncol. 2018;36:1668-74.

41. Betof Warner A, Palmer JS, Shoushtari AN, Goldman DA, Panageas KS, Hayes SA, et al. Long-term outcomes and responses to retreatment in patients with melanoma treated with PD-1 blockade. J Clin Oncol. 2020:JCO1901464. https://doi.org/10.1200/ JCO.19.01464.

42. Jansen YJL, Rozeman EA, Mason R, Goldinger SM, Geukes Foppen MH, Hoejberg L, et al. Discontinuation of anti-PD-1 antibody therapy in the absence of disease progression or treatment limiting toxicity: clinical outcomes in advanced melanoma. Ann Oncol. 2019;30:1154-61.

43. Tan AC, Emmett L, Lo S, Liu V, Kapoor R, Carlino MS, et al. FDGPET response and outcome from anti-PD-1 therapy in metastatic melanoma. Ann Oncol. 2018;29:2115-20.

Publisher's note Springer Nature remains neutral with regard to jurisdictional claims in published maps and institutional affiliations. 